\title{
The ending lamination space of the five-punctured sphere is the Nöbeling curve
}

\author{
Sebastian Hensel ${ }^{\alpha \circledast \text { \& }}$ \&iotr Przytycki抱 \\ ${ }^{a}$ Mathematisches Institut, Universität Bonn, \\ Endenicher Allee 60, 53115 Bonn, Germany \\ e-mail: lop lop@math . uni-bonn.de \\ ${ }^{b}$ Institute of Mathematics, Polish Academy of Sciences, \\ Sniadeckich 8, 00-956 Warsaw, Poland \\ e-mail:pprzytyc@mimuw.edu.pl
}

\begin{abstract}
We prove that the ending lamination space of the five-punctured sphere is homeomorphic to the Nöbeling curve.
\end{abstract}

\section{Introduction}

Let $S_{g, p}$ be the orientable surface of genus $g$ with $p$ punctures. The set of essential simple closed curves on the surface $S_{g, p}$ can be arranged into the curve complex $\mathcal{C}=\mathcal{C}\left(S_{g, p}\right)$. The combinatorics of this intricate object is used in particular to study the mapping class group of $S_{g, p}$. The curve complex is particularly useful in view of the result of Masur and Minsky [MM99, Theorem 1.1] which says that $\mathcal{C}$ is hyperbolic in the sense of Gromov. Hence, using the Gromov product (see [BH99, Chapter III.H]), one can define the Gromov boundary $\partial \mathcal{C}$ in the usual way. Note that the boundary $\partial \mathcal{C}$ is in general not compact, since the curve complex $\mathcal{C}$ is not locally finite. Hence the topology of $\partial \mathcal{C}$ can be a rich subject to explore.

Interestingly, the boundary $\partial \mathcal{C}$ arises naturally also from another construction. Namely, Klarreich [Kla99, Theorem 1.3] (see also [Ham06, Section 1]) proved that $\partial \mathcal{C}$ is homeomorphic, by an explicit homeomorphism, to the ending lamination space $\mathcal{E} \mathcal{L}=\mathcal{E} \mathcal{L}\left(S_{g, p}\right)$. To define this space, we need to recall some standard notions.

We denote the space of geodesic laminations on $S_{g, p}$ as usual by $\mathcal{L}=$ $\mathcal{L}\left(S_{g, p}\right)$. An ending lamination is a minimal filling geodesic lamination. The set of all ending laminations is denoted by $\mathcal{E} \mathcal{L} \subset \mathcal{L}$. It remains to describe the

\footnotetext{
*Supported by the Max Planck Institute for Mathematics in Bonn.

${ }^{\dagger}$ Partially supported by MNiSW grant N201 012 32/0718 and the Foundation for Polish Science.
} 
topology on the set $\mathcal{E} \mathcal{L}$. Let $\mathcal{P} \mathcal{M} \mathcal{L}$ denote the space of projective measured laminations. Let $\phi: \mathcal{P} \mathcal{M L} \rightarrow \mathcal{L}$ be the measure forgetting map which maps a projective measured lamination to its support. Note that $\phi$ is not surjective, because there are geodesic laminations which do not admit a transverse measure of full support. However, every ending lamination admits a transverse measure of full support, so $\mathcal{E} \mathcal{L}$ is contained in the image of $\phi$. Let $\mathcal{M P \mathcal { L }} \subset \mathcal{P} \mathcal{M L}$ be the preimage of $\mathcal{E} \mathcal{L}$ under $\phi$. The space $\mathcal{E} \mathcal{L}$ is naturally equipped with the topology induced from $\mathcal{M P \mathcal { M }}$ by the quotient $\operatorname{map} \phi$.

Here is a short account on what is known about the ending lamination space $\mathcal{E} \mathcal{L}$ depending on $g$ and $p$. We call $\xi=3 g-3+p$ the complexity of the surface. The cases where the complexity is at most one are easy and wellknown, in particular for $\xi=1$ (i.e. in the case where the surface is the fourpunctured sphere or the once-punctured torus) we have $\mathcal{E} \mathcal{L} \simeq \mathbb{R} \backslash \mathbb{Q}$. Assume now $\xi>1$, i.e. the surface in question is hyperbolic and non-exceptional. In this case Gabai Gab09] showed that $\mathcal{E} \mathcal{L}$ is connected, locally path-connected, and cyclic, which concluded a series of results in this direction. Previously Leininger-Schleimer [LS08] proved that $\mathcal{E} \mathcal{L}$ is connected, provided $g \geq 4$, or $g \geq 2$ and $p \geq 1$. Moreover, Leininger-Mj-Schleimer [LMS08] proved that if $g \geq 2$ and $p=1$, then $\mathcal{E} \mathcal{L}$ is locally path connected.

In spite of the fact that so little about $\mathcal{E} \mathcal{L}$ is known so far, we were encouraged by Mladen Bestvina to address the following.

Conjecture 1.1. The ending lamination space of $S_{g, p}$ is homeomorphic to the $(\xi-1)$-dimensional Nöbeling space.

Definition 1.2. The $m$-dimensional Nöbeling space $N_{m}^{2 m+1}$ is the topological space obtained from $\mathbb{R}^{2 m+1}$ by removing all points with at least $m+1$ rational coordinates.

In this terminology, the ending lamination space of the four-punctured sphere (and the once-punctured torus) is homeomorphic to the 0-dimensional Nöbeling space. This agrees with Conjecture 1.1.

The Nöbeling curve is the 1-dimensional Nöbeling space, i.e. the topological space obtained from $\mathbb{R}^{3}$ by removing all points with at least two rational coordinates. The main result of this article is to confirm Conjecture $1.1 \mathrm{in}$ the following case.

Theorem 1.3. The ending lamination space of the five-punctured sphere is homeomorphic to the Nöbeling curve.

Since $\mathcal{E} \mathcal{L}$ is homeomorphic to the Gromov boundary of the curve complex, which is the same (as a simplicial complex) for the twice-punctured torus and the five-punctured sphere (see [Lu00, Lemma 2.1(a)]), we have the following. 
Corollary 1.4. The ending lamination space of the twice-punctured torus is homeomorphic to the Nöbeling curve.

The article is organised as follows. In Section 2 we provide a topological characterisation of the Nöbeling curve which we use in the proof of Theorem 1.3. In Section 3, using train tracks, we choose a convenient neighbourhood basis for the ending lamination space. Then, in Section 4, we give an account on Gabai's method for constructing paths in $\mathcal{E} \mathcal{L}$.

The proof of Theorem 1.3 splits into two parts. In Section 5 we begin the proof and in particular we obtain a dimension bound. The main part of the proof is to obtain a universality property, with which we conclude in Section 6.

We thank Andrzej Nagórko for discussions on the Nöbeling curve and for the characterisation in Section 2, Ursula Hamenstädt for discussions on the ending lamination space and for suggestions on how to improve our preprint; and Mladen Bestvina and Saul Schleimer for encouragement. This work was partially carried out during the stay of the second author at the Hausdorff Institute of Mathematics in Bonn.

\section{The Nöbeling curve}

In this section we give a useful characterisation of the Nöbeling curve following from Kawamura-Levin-Tymchatyn [KLT97]. We learned about this characterisation and the way to derive it from [KLT97] using a standard topological argument from Andrzej Nagórko.

Theorem 2.1. If a Polish space of dimension 1 is connected, locally path connected and satisfies the locally finite 1-discs property, then it is homeomorphic to the Nöbeling curve.

Recall that a topological space is Polish if it is separable and admits a complete metric. A topological space has dimension 0 (resp. dimension at most $m$, for $m>0$ ) if each point has a basis of neighbourhoods with empty boundaries (resp. with boundaries of dimension at most $m-1$ ). A space has dimension $m$, for $m>0$, if it has dimension at most $m$, but does not have dimension $m-1$. In case of a Polish space this coincides with the usual covering dimension (see Eng78, Theorem 1.7.7]).

Definition 2.2. A topological space $X$ satisfies the locally finite $m$-discs property if we have the following. For any family of continuous maps $f_{n}: I^{m}=$ $[0,1]^{m} \rightarrow X$, where $n \in \mathbb{N}$, and any open cover $\mathcal{U}$ of $X$, there are continuous maps $g_{n}: I^{m} \rightarrow X$ such that 
(i) for each $x \in X$ there is a neighbourhood $U \ni x$ satisfying $g_{n}\left(I^{m}\right) \cap U=\emptyset$ for sufficiently large $n$,

(ii) for each $t \in I^{m}, n \in \mathbb{N}$, there is $U \in \mathcal{U}$ such that both $f_{n}(t)$ and $g_{n}(t)$ lie in $U$ (we say that such $f_{n}$ and $g_{n}$ are $\mathcal{U}$-close).

If additionally $g_{n}\left(I^{m}\right)$ may be required to be pairwise disjoint, then $X$ satisfies the discrete $m$-discs property.

In the remaining part of this section we explain how to derive Theorem 2.1 from the following.

Theorem 2.3 ([KLT97, Theorem 2.2]). A 1-dimensional Polish space is the Nöbeling curve if and only if it is an absolute extensor in dimension 1 and strongly universal in dimension 1.

In fact, in order to address Conjecture 1.1 in the future, we have decided to discuss the higher dimensional analogue of Theorem 2.3.

Theorem 2.4 ([Nag06, Topological rigidity theorem]). An m-dimensional Polish space is the m-dimensional Nöbeling space if and only if it is an absolute extensor in dimension $m$ and strongly universal in dimension $m$.

A metric space $X$ is an absolute extensor in dimension $m$, if every continuous map into $X$ from a closed subset of an at most $m$-dimensional metric space extends over the entire space. Assume now that $X$ is locally $k-$ connected for every $k<m$ (see [Dug58, Definition 3.1], for $m=1$ this means that $X$ is locally path connected). In that case, by Dugundji Dug58, Theorem 9.1], $X$ is an absolute extensor in dimension $m$ if and only if all of its homotopy groups in dimension less than $m$ vanish. For $m=1$ this means that $X$ is connected. Summarizing, if a metric space is locally $k-$ connected for every $k<m$, and all of its homotopy groups in dimension less than $m$ vanish, then it is an absolute extensor in dimension $m$. In particular, if a metric space is connected and locally path connected, then it is an absolute extensor in dimension 1.

A Polish space $X$ is strongly universal in dimension $m$ if any continuous map $f: Y \rightarrow X$ from an at most $m$-dimensional Polish space $Y$ to $X$ is approximable by closed embeddings. This means that for any open cover $\mathcal{U}$ of $X$ there is a closed embedding $g: Y \rightarrow X$ such that $f$ and $g$ are $\mathcal{U}$-close. We discuss below, under what hypothesis strong universality in dimension $m$ follows from the locally finite $m$-discs property.

By [Cur85, discussion after Theorem 2.4], any Polish space $X$ satisfying the locally finite $m$-discs property satisfies also the discrete $m$-discs property. Bowers [Bow85, Theorem in Appendix, part (2)] proves that the latter 
implies strong universality in dimension $m$, under the hypothesis that $X$ is an ANR. Recall that a topological space $X$ is an absolute neighbourhood retract (shortly, an $A N R$ ) if for each closed subset $A \subset X$, which is normal, there is an open neighbourhood $U \subset X$ such that $A$ is a retract of $U$. Unfortunately, Nöbeling spaces are not ANR, hence Bowers' theorem as stated is not sufficient for our purposes. However, his proof yields the following.

Theorem 2.5. Let $X$ be a Polish space which is locally $k$-connected for all $k<m$. If $X$ satisfies the discrete $m$-discs property, then it is strongly universal in dimension $m$.

In other words, we can replace the ANR hypothesis in Bow85, Theorem in Appendix] by local $k$-connectedness for all $k<m$. Indeed, the only two places in the proof, where the ANR hypothesis is used, are lines 1 and 5 on page 129, in the proof of Lemma C. However, in both cases the argument only requires the following property (which is satisfied if $X$ is an ANR). Namely, let $k<m$ and let $S^{k}$ be the $k$-sphere. Bowers' argument requires that for every open cover $\mathcal{U}$ of $X$, there is a refinement $\mathcal{U}^{\prime}$, such that if $f_{0}, f_{1}: S^{k} \rightarrow X$ are $\mathcal{U}^{\prime}$-close, then there is a homotopy between $f_{0}$ and $f_{1}$ with each track contained in some $U \in \mathcal{U}$. By Dug58, Theorem 5.1] this property follows from local $k$-connectedness. This concludes the argument for Theorem 2.5.

By Theorems 2.4 and 2.5 and by the preceding discussion we conclude with the following, which in the case of $m=1$ amounts exactly to Theorem 2.1 .

Corollary 2.6. Let $X$ be Polish space of dimension $m$ which is locally $k-$ connected for every $k<m$, and all of whose homotopy groups in dimension less than $m$ vanish. Assume that $X$ satisfies the locally finite $m$-discs property. Then $X$ is homeomorphic to the $m$-dimensional Nöbeling space.

\section{Train track partitions}

Our strategy of proving Theorem 1.3 is to use the topology of $\mathcal{P} \mathcal{M L}$, the space of projective measured laminations, to obtain information about the topology of the ending lamination space $\mathcal{E} \mathcal{L}$. To this end, we construct a sequence of finer and finer partitions of $\mathcal{P} \mathcal{M L}$ into polyhedra using Thurston's notion of train tracks (see [Thu80, Section 8.9]). We then show that these polyhedra project to a convenient neighbourhood basis of $\mathcal{E} \mathcal{L}$.

For a thorough treatment of train tracks, as well as the basic definitions, we refer the reader to the book of Penner and Harer [PH92]. Note however that, in contrast to the treatment in [PH92, for us every train track is generic 
(i.e. each switch is at most trivalent). In the following, we briefly recall some definitions and statements important to the current work.

Let $\tau$ be a recurrent train track. We denote by $P(\tau)$ the polyhedron of projective measures of $\tau$, that is the set of all projective measured laminations which are carried by $\tau . P(\tau)$ has the structure of an affine polyhedron, where the faces of $P(\tau)$ correspond to recurrent proper subtracks $\tau^{\prime}<\tau$ (see [PH92, pp. 116-117]). The inclusion map $P(\tau) \subset \mathcal{P} \mathcal{M L}$, where $P(\tau)$ is equipped with the topology coming from the polyhedral structure, is continuous. In particular, for any train track $\tau$ the polyhedron of projective measures $P(\tau)$ is a closed set in $\mathcal{P} \mathcal{M L}$. The interior of $P(\tau)$ is its interior with respect to the polyhedral structure, i.e. the set of transverse measures which put positive mass on each branch of $\tau$. Note that in general this is not the interior of the set $P(\tau) \subset \mathcal{P} \mathcal{M L}$ with respect to the topology of $\mathcal{P} \mathcal{M L}$. In the sequel we denote the interior of the polyhedron of projective measures by $V(\tau) \subset \mathcal{P} \mathcal{M L}$. We denote the boundary $P(\tau) \backslash V(\tau)$ of the polyhedron of projective measures by $\partial V(\tau)$. From now on, the expression boundary of $X$ will always mean $\operatorname{Fr} X=\bar{X} \backslash \operatorname{int} X$ (the boundary in the topological sense). Note that in this terminology $\partial V(\tau)$ might not be the boundary of $V(\tau) \subset \mathcal{P} \mathcal{M L}$. Let

$$
U(\tau)=\phi(V(\tau) \cap \mathcal{M P} \mathcal{M} \mathcal{L})
$$

(equivalently, $U(\tau)$ is the set of ending laminations which are fully carried by $\tau$ ). We denote the inverse correspondence between (families of) these sets by $\Psi$, i.e. $\Psi(U(\tau))=V(\tau)$.

Unless stated otherwise, from now on we restrict to the case of $S_{0,5}$, where $\mathcal{P} \mathcal{M L}$ is 3 -dimensional. We call a train track $\eta$ complete if it is recurrent, transversely recurrent and maximal. Recall that if $\eta$ is complete, then $V(\eta)$ is 3-dimensional, hence open in $\mathcal{P} \mathcal{M L}$ (see e.g. [PH92, Lemma 3.1.2]) and consequently $U(\eta)$ is open in $\mathcal{E} \mathcal{L}$. In particular we have $\partial V(\eta)=\operatorname{Fr} V(\eta)$. We call a train track $\sigma$ nearly complete, if it is birecurrent, carries an ending lamination and $P(\sigma)$ is 2-dimensional (in particular $\sigma$ is not complete).

Remark 3.1. Let $\mu_{0}, \mu_{1}$ be measured geodesic laminations which do not intersect transversally. Suppose that for some train track $\tau$ its polyhedron of projective measures $P(\tau)$ contains a projective class of $\mu_{t}=(1-t) \mu_{0}+t \mu_{1}$ for some $t \neq 0,1$. Then the whole interval $\left\{\mu_{t}\right\}_{t \in[0,1]}$ projects into $P(\tau)$. This is because the support of $\mu_{t}$ equals $\phi\left(\mu_{1}\right) \cup \phi\left(\mu_{2}\right)$ except maybe for $t=0$ or 1 , and projective measured laminations are carried by train tracks if and only if their supports are.

We will need the following lemma, which shows how $\mathcal{M P \mathcal { M }} \mathcal{L}$ can intersect the polyhedron of projective measures of a complete or nearly complete train track. 


\section{Lemma 3.2.}

(i) Let $\sigma$ be a nearly complete train track. Then $\partial V(\sigma)$ contains no filling lamination. In particular, $\partial V(\sigma)$ is disjoint from $\mathcal{M P \mathcal { M L }}$.

(ii) Let $\eta$ be a complete train track. Then the 1-skeleton of $\partial V(\eta)$ contains no filling lamination. In particular, the intersection of the 1-skeleton of $\partial V(\eta)$ with $\mathcal{M P \mathcal { M }} \mathcal{L}$ is empty.

(iii) Let $\sigma$ be a nearly complete train track. Then $U(\sigma)$ is closed in $\mathcal{E} \mathcal{L}$.

\section{Proof.}

(i) Let $\sigma$ be a nearly complete train track. Recall that $\partial V(\sigma)$ is the union of $P(\tau)$ over all recurrent proper subtracks $\tau<\sigma$. We show that no proper subtrack of $\sigma$ is filling, which immediately implies assertion (i).

Since $\sigma$ is nearly complete, it carries a filling lamination. Hence its complementary regions are topological discs or once-punctured discs. Thus, on a five-punctured sphere a nearly complete train track has at least five complementary regions. Each of those regions gives a contribution of at least $-\frac{1}{2}$ to the (generalised) Euler characteristic of $S_{0,5}$, with a contribution of exactly $-\frac{1}{2}$ if and only if the component is a triangle or a once-punctured monogon.

If $\sigma$ had more than five complementary regions, the fact that $\chi\left(S_{0,5}\right)$ equals -3 would imply that these regions have to be five once-punctured monogons and one triangle - which would mean that $\sigma$ was complete.

Hence, $\sigma$ has four once-punctured monogons and one once-punctured bigon as complementary regions. A proper subtrack $\tau<\sigma$ needs to erase at least one branch of $\sigma$, and hence join two of these regions (or one to itself). Thus some complementary region of $\tau$ contains either two punctures or an essential curve - hence $\tau$ is not filling.

(ii) Let $\eta$ be complete. The 1 -skeleton of $\partial V(\eta)$ is the union of $P(\tau)$ over recurrent $\tau<\eta$ which are obtained from $\eta$ by removing at least two branches. Now assertion (ii) follows with the same Euler characteristic argument as in the proof of assertion (i).

(iii) Let $\sigma$ be a nearly complete train track. The polyhedron of projective measures $P(\sigma)$ is a closed set in $\mathcal{P} \mathcal{M L}$. Since the topology of $\mathcal{E} \mathcal{L}$ is

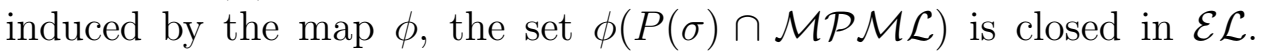
However, by assertion (i), we have $P(\sigma) \cap \mathcal{M P} \mathcal{M L}=V(\sigma) \cap \mathcal{M P} \mathcal{M} \mathcal{L}$ and hence $U(\sigma)=\phi(V(\sigma) \cap \mathcal{M P \mathcal { M } L})$ is closed in $\mathcal{E} \mathcal{L}$. 
In the remaining part of this section, our aim is to find a convenient neighbourhood basis for $\mathcal{E} \mathcal{L}$, determined by a certain set of train tracks.

Definition 3.3. Let $\mathrm{T}$ be a finite collection of complete train tracks and let $\Sigma$ be a finite collection of nearly complete train tracks. The pair $(\mathrm{T}, \Sigma)$ is called a train track partition if all $V(\tau)$ are pairwise disjoint, for $\tau \in \mathrm{T} \cup \Sigma$, and together cover all of $\mathcal{M P \mathcal { M }}$.

Note that in particular all $U(\tau)$ are pairwise disjoint, for $\tau \in \mathrm{T} \cup \Sigma$, and cover all of $\mathcal{E} \mathcal{L}$.

\section{Examples 3.4.}

(i) Let $P$ be any pants decomposition for $S_{0,5}$. Let $\mathrm{T}$ be the set of complete standard train tracks with respect to $P$ (see [PH92, Section 2.6]) and let $\Sigma$ be the set of their nearly complete subtracks.

We claim that $(T, \Sigma)$ is a train track partition. To this end, first note that the $P(\eta)$, for $\eta \in \mathrm{T}$, cover all of $\mathcal{P} \mathcal{M L}$ and each projective measured lamination $\lambda$ is fully carried by a unique subtrack $\tau$ of one of the $\eta \in \mathrm{T}$ (see [PH92, Sections 2.7 and 2.8]). In particular, $V(\tau)$ are

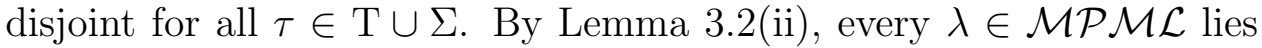
in $V(\tau)$ for some $\tau \in \mathrm{T} \cup \Sigma$.

We call such a pair $(\mathrm{T}, \Sigma)$ a standard train track partition.

(ii) Let $(\mathrm{T}, \Sigma)$ be a train track partition, and let $\eta \in T$ be a complete train track. Denote by $\eta_{L}$ (and $\eta_{R}$ ) the left (respectively right) split of $\eta$ along some large branch $b$. Note that splitting $\eta$ amounts to cutting $P(\eta)$ along a hyperplane, so that we have $P(\eta)=P\left(\eta_{L}\right) \cup P\left(\eta_{R}\right)$ and $P\left(\eta_{L}\right) \cap P\left(\eta_{R}\right)=P(\sigma)$ for a common subtrack $\sigma$ of $\eta_{L}$ and $\eta_{R}$.

If both $\eta_{L}$ and $\eta_{R}$ are complete, define $\mathrm{T}^{\prime}$ by replacing $\eta \in T$ by $\left\{\eta_{L}, \eta_{R}\right\}$. Then, if $\sigma$ is nearly complete, add $\sigma$ to $\Sigma$ to obtain $\Sigma^{\prime}$. If only one of the two train tracks $\eta_{L}$ and $\eta_{R}$ is complete, replace $\eta \in \mathrm{T}$ by this train track to get $\mathrm{T}^{\prime}$ and set $\Sigma^{\prime}=\Sigma$.

Note that in both cases the resulting pair $\left(\mathrm{T}^{\prime}, \Sigma^{\prime}\right)$ is a train track partition. We say that $\left(\mathrm{T}^{\prime}, \Sigma^{\prime}\right)$ is obtained from $(\mathrm{T}, \Sigma)$ by a complete splitting move along $b$.

(iii) Let $(\mathrm{T}, \Sigma)$ be a train track partition. Let $\sigma \in \Sigma$ be any nearly complete train track. Consider the left (respectively right) split $\sigma_{L}$ (and $\sigma_{R}$ ) along some large branch $b$. As above we have $P(\sigma)=P\left(\sigma_{L}\right) \cup P\left(\sigma_{R}\right)$ and $P\left(\sigma_{L}\right) \cap P\left(\sigma_{R}\right)=P(\tau)$ for a common subtrack $\tau$ of $\sigma_{L}$ and $\sigma_{R}$. 
If both $\sigma_{L}$ and $\sigma_{R}$ are nearly complete, define $\Sigma^{\prime}$ by replacing $\sigma \in \Sigma$ by $\left\{\sigma_{L}, \sigma_{R}\right\}$. Otherwise, replace $\sigma$ by the train track $\sigma_{L}$ or $\sigma_{R}$ which is nearly complete. Note that in both cases, by Lemma 3.2(i), the resulting pair $\left(\mathrm{T}, \Sigma^{\prime}\right)$ is a train track partition. We say that $\left(\mathrm{T}, \Sigma^{\prime}\right)$ is obtained from $(\mathrm{T}, \Sigma)$ by a nearly complete splitting move along $b$.

We now use the above examples to obtain the following.

Theorem 3.5. There exists a sequence $\mathcal{S}=\left(\left(\mathrm{T}_{k}, \Sigma_{k}\right)\right)_{k=0}^{\infty}$ of train track partitions satisfying the following two properties.

(Subdivision) Let $K \geq k \geq 0$. For each $\eta \in \mathrm{T}_{K}$ there is an $\eta^{\prime} \in \mathrm{T}_{k}$ satisfying $V(\eta) \subset V\left(\eta^{\prime}\right)$. For each $\sigma \in \Sigma_{K}$ there is a $\tau \in \mathrm{T}_{k} \cup \Sigma_{k}$ satisfying $V(\sigma) \subset V(\tau)$.

(Fineness) For each ending lamination $\lambda$ and each open set $W$ in $\mathcal{P} \mathcal{M L}$ containing $\phi^{-1}(\lambda)$ there is an open set $V$ in $\mathcal{P} \mathcal{M L}$ satisfying $W \supset V \supset$ $\phi^{-1}(\lambda)$ of the following form.

Either $V=V(\eta)$ with $\eta \in \mathrm{T}_{k}$, for some $k \geq 0$, or $V=V\left(\eta_{1}\right) \cup V(\sigma) \cup$ $V\left(\eta_{2}\right)$ with $\eta_{i} \in \mathrm{T}_{k_{i}}, \sigma \in \Sigma_{k}$, for some $k_{1}, k_{2}, k \geq 0$. In the latter case we additionally require $P(\sigma) \subset \partial V\left(\eta_{1}\right) \cap \partial V\left(\eta_{2}\right)$ (see Figure 1).

$W$ denote by $\mathcal{V}(\mathcal{S})$ the family of all open sets $V$ of above type.
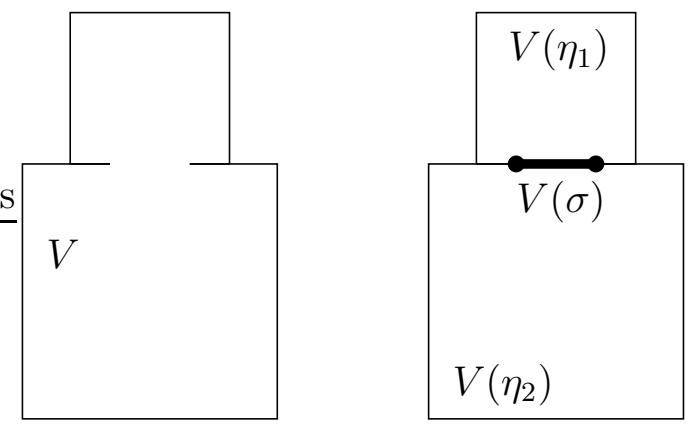

Figure 1: The case where $V=V\left(\eta_{1}\right) \cup V(\sigma) \cup V\left(\eta_{2}\right)$; we draw $V(\sigma)$ thick just to emphasise its presence; note that for simplicity the configuration is depicted in dimension 2 instead of 3.

Before we begin the proof of Theorem 3.5, we record the following.

Remark 3.6. If we have a sequence of train track partitions so that each $\left(\mathrm{T}_{k+1}, \Sigma_{k+1}\right)$ is obtained from $\left(\mathrm{T}_{k}, \Sigma_{k}\right)$ by a complete splitting move or a nearly complete splitting move (see Examples 3.4(ii,iii)), then they satisfy property (Subdivision). Moreover, property (Subdivision) is preserved under passing to a subsequence. 
Definition 3.7. We call sequences $\mathcal{S}=\left(\left(\mathrm{T}_{k}, \Sigma_{k}\right)\right)_{k=0}^{\infty}$ satisfying (Subdivision) and (Fineness) good partition sequences. In this terminology Theorem 3.5 says that there exists a good partition sequence.

Remark 3.8. If $\left(\left(\mathrm{T}_{k}, \Sigma_{k}\right)\right)_{k=0}^{\infty}$ is a good partition sequence, then for any $K \geq 0$ the sequence $\left(\left(\mathrm{T}_{k}, \Sigma_{k}\right)\right)_{k=K}^{\infty}$ is also a good partition sequence.

Properties (Subdivision) and (Fineness) have the following immediate consequences for the sets $U(\tau)$ in the ending lamination space.

Corollary 3.9. Let $\mathcal{S}=\left(\left(\mathrm{T}_{k}, \Sigma_{k}\right)\right)_{k=1}^{\infty}$ be a good partition sequence. Then (Subdivision) holds after replacing each $V(\tau)$ with $U(\tau)$. Furthermore, let $\mathcal{U}(\mathcal{S})=\{\phi(V \cap \mathcal{M P \mathcal { M L }})\}_{V \in \mathcal{V}(\mathcal{S})}$. Then $\mathcal{U}(\mathcal{S})$ is a neighbourhood basis of $\mathcal{E} \mathcal{L}$

We denote by $\Psi: \mathcal{U}(\mathcal{S}) \rightarrow \mathcal{V}(\mathcal{S})$ the map extending the map $\Psi(U(\eta))=$ $V(\eta)$ by $\Psi\left(U\left(\eta_{1}\right) \cup U(\sigma) \cup U\left(\eta_{2}\right)\right)=\Psi\left(U\left(\eta_{1}\right)\right) \cup \Psi(U(\sigma)) \cup \Psi\left(U\left(\eta_{2}\right)\right)$.

The remaining part of this section is devoted to the proof of Theorem 3.5 . We need to recall some facts about full splitting sequences.

Let $b_{1}, \ldots, b_{l}$ be the large branches of a train track $\tau$. Note that, if $\tau^{\prime}$ is obtained from $\tau$ by a split at $b_{i}$, every $b_{j}$ is still a large branch of $\tau^{\prime}$ for $j \neq i$. A full split of $\tau$ (see [Ham09, Section 5]) is a train track which is obtained from $\tau$ by splitting at each large branch $b_{i}$ exactly once (we also say that this train track is obtained from $\tau$ by a full split). A full splitting sequence is a sequence of train tracks $\left(\tau^{i}\right)_{i}$ such that $\tau^{n+1}$ is obtained from $\tau^{n}$ by a full split. For an ending lamination $\lambda$ carried by $\tau$, a full $\lambda$-splitting sequence of $\tau$ is a full splitting sequence $\left(\tau^{i}\right)_{i}$ with $\tau^{0}=\tau$ and such that each $\tau^{i}$ carries $\lambda$.

The following immediate consequence of [Mos03, Theorem 8.5.1] is the central part of the upcoming proof of Theorem 3.5. (A similar theorem is obtained in [Ham09.)

Theorem 3.10. Let $\lambda$ be an ending lamination and let $\left(\tau^{i}\right)_{i}$ be a full $\lambda-$ splitting sequence of some train track $\tau$. Then we have

$$
\bigcap_{i=1}^{\infty} P\left(\tau^{i}\right)=\phi^{-1}(\lambda) \text {. }
$$

In particular, for any open neighbourhood $W$ of $\phi^{-1}(\lambda)$ in $\mathcal{P} \mathcal{M L}$, there is some $i_{0}>0$ such that for all $i>i_{0}$ we have $P\left(\tau^{i}\right) \subset W$.

Proof of Theorem 3.5. Let $P$ be a pants decomposition for $S_{0,5}$ and let $\left(\mathrm{T}_{0}, \Sigma_{0}\right)$ be the associated standard train track partition. We now describe an inductive procedure for building $\left(\mathrm{T}_{k}, \Sigma_{k}\right)$, where $k \geq 0$, which will satisfy property (Subdivision) and the following two additional properties. 


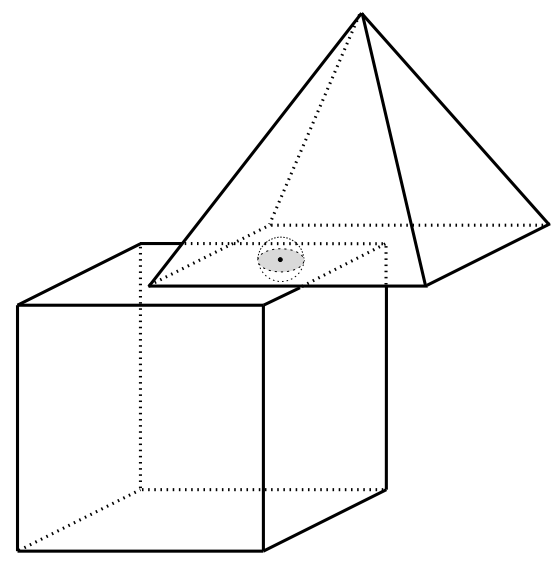

Figure 2: The open neighbourhood as in property (Adjacency).

(Adjacency) If $\mu \in \mathcal{M P M L}$ lies in $V(\sigma)$, where $\sigma \in \Sigma_{k}$ for some $k \geq 0$, then there are $\eta \neq \eta^{\prime} \in \mathrm{T}_{k}$ such that $\mu$ lies in $P(\eta) \cap P\left(\eta^{\prime}\right)$. Moreover, the set obtained from

$$
V(\eta) \cup V\left(\eta^{\prime}\right) \cup\left(\partial V(\eta) \cap \partial V\left(\eta^{\prime}\right)\right)
$$

by removing the 1 -skeleta of $P(\eta)$ and $P\left(\eta^{\prime}\right)$ is an open neighbourhood of $\mu$ (see Figure 2).

(Full splits) If $\tau \in \mathrm{T}_{k} \cup \Sigma_{k}$ for some $k \geq 0$ and $\tau^{\prime}$ is a complete or nearly complete train track obtained from $\tau$ by a full split, then $\tau^{\prime}$ belongs to $\mathrm{T}_{k+1} \cup \Sigma_{k+1}$.

Note that the standard train track partition $\left(\mathrm{T}_{0}, \Sigma_{0}\right)$ satisfies property (Adjacency). Moreover, by Lemma 3.2(ii), a train track partition obtained by a complete splitting move or a nearly complete splitting move (see Examples 3.4(ii,iii)) from another train partition satisfying property (Adjacency), satisfies property (Adjacency) itself.

We now describe our inductive procedure. Suppose that the train track partition $\left(\mathrm{T}_{k}, \Sigma_{k}\right)$ has already been defined. Roughly speaking we now perform the following operation. For each $\eta \in \mathrm{T}_{k}$ we use complete splitting moves along all large branches of $\eta$ to obtain $\left(\mathrm{T}_{k}^{\prime}, \Sigma_{k}^{\prime}\right)$. In the second step we perform nearly complete splitting moves for each $\sigma \in \Sigma_{k}$ along all large branches of $\sigma$ to obtain $\left(\mathrm{T}_{k+1}, \Sigma_{k+1}\right)$.

More precisely, let $\eta \in \mathrm{T}_{k}$ be a complete train track. Denote the large branches of $\eta$ by $b_{1}, \ldots, b_{l}$. We now perform a complete splitting move along $b_{1}$ to obtain a new partition $\left(\mathrm{T}_{k}^{1}, \Sigma_{k}^{1}\right)$. The set $\mathrm{T}_{k}^{1}$ contains one or two train 
tracks corresponding to the two possible splits of $\eta$ along $b_{1}$. Each of those still contains $b_{2}, \ldots, b_{l}$ as large branches. We perform complete splitting moves along $b_{2}$ for those (one or two) train tracks in $\mathrm{T}_{k}^{1}$ to obtain the partition $\left(\mathrm{T}_{k}^{2}, \Sigma_{k}^{2}\right)$. The set $\mathrm{T}_{k}^{2}$ contains now up to four train tracks corresponding to the possible splits of $\eta$ along $b_{1}$ and $b_{2}$. We split all these (up to four) train tracks along $b_{3}$ and continue this way for all large branches $b_{1}, \ldots, b_{l}$ until we terminate with $\left(\mathrm{T}_{k}^{l}, \Sigma_{k}^{l}\right)$.

Note that this partition now contains every full split $\eta^{1}$ of $\eta$, if $\eta^{1}$ is a complete train track. Moreover, we have $\mathrm{T}_{k} \backslash\{\eta\} \subset \mathrm{T}_{k}^{l}$. We now repeat the same procedure for all $\eta^{\prime} \in \mathrm{T}_{k} \backslash\{\eta\}$. The resulting partition $\left(\mathrm{T}_{k}^{\prime}, \Sigma_{k}^{\prime}\right)$ contains for every $\eta \in \mathrm{T}_{k}$ all of its full splits which are complete.

In the second step, we obtain $\left(\mathrm{T}_{k+1}, \Sigma_{k+1}\right)$ from $\left(\mathrm{T}_{k}^{\prime}, \Sigma_{k}^{\prime}\right)$ by performing the analogous operation with all elements of $\Sigma_{k} \subset \Sigma_{k}^{\prime}$. Note that $\mathrm{T}_{k+1}=\mathrm{T}_{k}^{\prime}$. This completes the definition of $\mathcal{S}=\left(\left(\mathrm{T}_{k}, \Sigma_{k}\right)\right)_{k=0}^{\infty}$.

Since all $\left(\mathrm{T}_{k}, \Sigma_{k}\right)$ are obtained from $\left(\mathrm{T}_{0}, \Sigma_{0}\right)$ by a sequence of complete splitting moves and nearly complete splitting moves, $\mathcal{S}$ satisfies property (Adjacency). By Remark [3.6, $\mathcal{S}$ satisfies also property (Subdivision). Furthermore, by construction, $\mathcal{S}$ satisfies property (Full splits). We now use this information to derive property (Fineness).

Let $\lambda \in \mathcal{E} \mathcal{L}$, and let $W \subset \mathcal{P} \mathcal{M L}$ be any open set containing $\phi^{-1}(\lambda)$. Let $\left(\eta^{i}\right)_{i}$ be a full $\lambda$-splitting sequence of some $\eta^{0} \in \mathrm{T}_{0}$ carrying $\lambda$. By property (Full splits), we have $\eta^{k} \in \mathrm{T}_{k}$. By Theorem [3.10, for sufficiently large $k$ we have $V\left(\eta^{k}\right) \subset W$. Hence if $\phi^{-1}(\lambda) \subset V\left(\eta^{k}\right)$, we are done. Otherwise, $\phi^{-1}(\lambda)$ is contained in $\partial V\left(\eta^{k}\right)$.

Then the strategy is roughly speaking the following. We consider the polyhedron $P\left(\eta^{\prime k}\right)$ "on the other side" of the face of $P\left(\eta^{k}\right)$ containing $\phi^{-1}(\lambda)$. We then split $\eta^{\prime k}$ until $P\left(\eta^{\prime K}\right)$ is contained in $W$. The face of $P\left(\eta^{\prime K}\right)$ containing $\phi^{-1}(\lambda)$ might not itself be a train track occurring in our partition sequence. However, there is some nearly complete train track $\sigma^{K} \in \Sigma^{K}$ carrying $\lambda$. We split $\sigma^{K}$ until its polyhedron of projective measures lies in the interior of the appropriate faces of both $P\left(\eta^{K}\right)$ and $P\left(\eta^{\prime K}\right)$. Then the resulting three train tracks define the required neighbourhood.

More precisely, by property (Adjacency), there is $\eta^{\prime k} \in \mathrm{T}_{k}$ with $\phi^{-1}(\lambda) \subset$ $P\left(\eta^{k}\right) \cap P\left(\eta^{\prime k}\right)$. By Theorem 3.10 and property (Full splits), there are some $K \geq k$ and $\eta^{\prime K} \in \mathrm{T}_{K}$ with $\phi^{-1}(\lambda) \subset P\left(\eta^{\prime K}\right) \subset W$. Let $\sigma^{K} \in \Sigma_{K}$ be the nearly complete train track carrying $\lambda$. By property (Adjacency), the set $N$ obtained from

$$
V\left(\eta^{K}\right) \cup V\left(\eta^{\prime K}\right) \cup\left(\partial V\left(\eta^{K}\right) \cap \partial V\left(\eta^{\prime K}\right)\right)
$$

by removing the 1 -skeleta of $P\left(\eta^{K}\right)$ and $P\left(\eta^{\prime K}\right)$, is an open neighbourhood 
of $\phi^{-1}(\lambda)$.

By Theorem 3.10 and property (Full splits), there is some $L \geq 0$ and $\sigma^{L} \in$ $\Sigma_{L}$ with $\phi^{-1}(\lambda) \subset P\left(\sigma^{L}\right) \subset N$. By Lemma 3.2(i), we have $\phi^{-1}(\lambda) \subset V\left(\sigma^{L}\right)$. We put $V=V\left(\eta^{K}\right) \cup V\left(\sigma^{L}\right) \cup V\left(\eta^{\prime K}\right)$. Then we have $\phi^{-1}(\lambda) \subset V \subset W$.

Since $P\left(\sigma^{L}\right)$ is 2-dimensional in $\partial V\left(\eta^{K}\right)$ and $\partial V\left(\eta^{\prime K}\right)$, we have that $V\left(\sigma^{L}\right)$ is open in $\partial V\left(\eta^{K}\right) \cap \partial V\left(\eta^{\prime K}\right)$. Hence each point of $V\left(\sigma^{L}\right)$ lies in the interior of $V$, and we conclude that $V$ is open, as desired.

\section{Almost filling paths}

In this section we give some account on Gabai's method of constructing paths in $\mathcal{E} \mathcal{L}$. This discussion is valid for any surface $S_{g, p}$ with $\xi=3 g-3+p \geq 2$. Gabai's main result is the following.

Theorem 4.1 (Gab09, Theorem 0.1]). $\mathcal{E} \mathcal{L}$ is connected, path connected and cyclic.

Here we give some details on Gabai's construction, which we need in the proof of Theorem 1.3. Recall [Gab09] that a geodesic lamination $\lambda$ is almost minimal almost filling if it has the form $\lambda=\lambda^{*} \cup \gamma$ where $\lambda^{*}$ has no isolated leaves, the closed (with respect to the path metric) complement of $\lambda^{*}$ supports at most one simple closed geodesic, and $\gamma$ is either this geodesic or is empty. We denote by $\mathcal{A M \mathcal { L }} \supset \mathcal{E} \mathcal{L}$ the set of all almost minimal almost filling geodesic laminations.

Gabai uses $P L$ almost filling paths $h: I=[0,1] \rightarrow \mathcal{P} \mathcal{M L}$ satisfying $\phi(h(t)) \in \mathcal{A M L}, \phi(h(0)), \phi(h(1)) \in \mathcal{E} \mathcal{L}$ and some additional properties satisfied by generic PL paths. We do not recall these properties, since we use only the combination of the following results. We assume that $\mathcal{P} \mathcal{M L}$ is equipped with a fixed metric, and we say that two points in $\mathcal{P} \mathcal{M L}$ are $\varepsilon-$ close if they are distance at most $\varepsilon$ in this metric.

Lemma 4.2 (Gab09, Lemma 2.9]). Let $h: I \rightarrow \mathcal{P M L}$ be a path with $\phi(h(0)), \phi(h(1)) \in \mathcal{E} \mathcal{L}$. Then for any $\varepsilon>0$ there is a $P L$ almost filling path $h^{\prime}: I \rightarrow \mathcal{P} \mathcal{M L}$ with the same endpoints and such that $h^{\prime}(t)$ is $\varepsilon$-close to $h(t)$, for all $t \in I$.

We now fix a hyperbolic metric on $S_{g, p}$ and consider geodesic laminations as subsets of the projective tangent bundle of $S_{g, p}$. The hyperbolic metric on $S_{g, p}$ induces a natural (Sasaki) metric on the projective tangent bundle. For a geodesic lamination $\lambda$, we denote by $N_{\varepsilon}^{P T}(\lambda)$ its $\varepsilon$-neighbourhood in this metric. The key element of the proof of Theorem 4.1 is the following crucial result. 
Lemma 4.3 (Gab09, Lemma 5.1]). If $h: I \rightarrow \mathcal{P M L}$ is a PL almost filling path, $\varepsilon>0, \delta>0$, then there exists a path $g: I \rightarrow \mathcal{E} \mathcal{L}$ with $g(0)=$ $\phi(h(0)), g(1)=\phi(h(1))$ such that for each $t \in[0,1]$ there exists $s \in I$ with $|s-t|<\delta$ satisfying

$$
h(s)^{*} \subset N_{\varepsilon}^{P T}(\tilde{g}(t)),
$$

for some diagonal extension $\tilde{g}(t)$ of $g(t)$.

We also need the following lemma, which roughly says that for $h$ and $g$ as in the assertion of Lemma 4.3, the preimage $\phi^{-1}(g(I))$ is not far away from $h(I)$ in $\mathcal{P} \mathcal{M L}$. We restrict to the case of the five-punctured sphere (although a version of this result is true in general). This way we may choose a good partition sequence $\mathcal{S}$ (see Definition 3.7). We denote by $\mathcal{V}(\mathcal{S}), \mathcal{U}(\mathcal{S})$ its associated families of open sets in $\mathcal{P} \mathcal{M L}$ and $\mathcal{E} \mathcal{L}$, respectively (see Theorem 3.5 and Corollary [3.9).

Lemma 4.4. Let $\lambda_{0} \in U \in \mathcal{U}(\mathcal{S})$. Then there is $U^{\prime} \in \mathcal{U}(\mathcal{S})$ with $\lambda_{0} \in U^{\prime}$ and $\varepsilon>0$ satisfying the following.

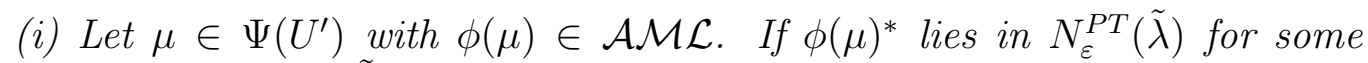
diagonal extension $\tilde{\lambda}$ of $\lambda \in \mathcal{E} \mathcal{L}$, then $\lambda \in U$.

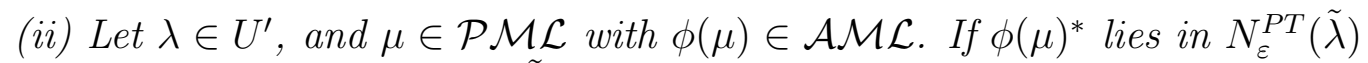
for some diagonal extension $\tilde{\lambda}$ of $\lambda$, then $\mu \in \Psi(U)$.

Proof. Part (i) is proved in course of the proof of [Gab09, Theorem 6.1] (local path connectedness of $\mathcal{E} \mathcal{L}$ ).

For part (ii), let $V=\Psi(U)$. By Theorem 3.5(Fineness), there are neighbourhoods $V_{1}, V_{2}, V^{\prime} \in \mathcal{V}(\mathcal{S})$ of $\phi^{-1}\left(\lambda_{0}\right)$ satisfying $\overline{V_{1}} \subset V, \bar{V}_{2} \subset V_{1}$, and $\bar{V}^{\prime} \subset V_{2}$ (see Figure 3). We prove that $U^{\prime}=\phi\left(V^{\prime} \cap \mathcal{M P \mathcal { M }} \mathcal{L}\right)$ satisfies the assertion of the lemma.

First we claim that if we have $\mu \in \mathcal{P} \mathcal{M L} \backslash V$ with $\phi(\mu) \in \mathcal{A M} \mathcal{L}$, then there is a projective measured lamination $\nu \in \mathcal{P} \mathcal{M L} \backslash V_{1}$ with support $\phi(\mu)^{*}$. Indeed, if $\phi(\mu)$ is an ending lamination, then we can take $\nu=\mu$. Otherwise we have $\phi(\mu)=\phi(\mu)^{*} \cup \gamma$ for some simple closed geodesic $\gamma$. Let $\nu$ be the projective measured lamination with support $\phi(\mu)^{*}$ obtained by restricting the measure $\mu$ to $\phi(\mu)^{*}$. By Remark 3.1, the interval of projective measured laminations determined by $\nu$ and $\gamma$ is contained in $\mathcal{P} \mathcal{M L} \backslash V_{1}$. This justifies the claim.

By Remark 3.1, the supports of any pair of projective measured laminations in $\bar{V}^{\prime}$ and $\mathcal{P} \mathcal{M} \mathcal{L} \backslash V_{1}$ intersect transversally. Observe that $\bar{V}^{\prime}$ and $\mathcal{P} \mathcal{M L} \backslash$ $V_{1}$ are compact in $\mathcal{P} \mathcal{M L}$. By super convergence of supports (Gab09, Proposition 3.2(i)]), there is $\delta>0$ satisfying the following. For any $\lambda \in \phi\left(\bar{V}^{\prime} \cap\right.$ 


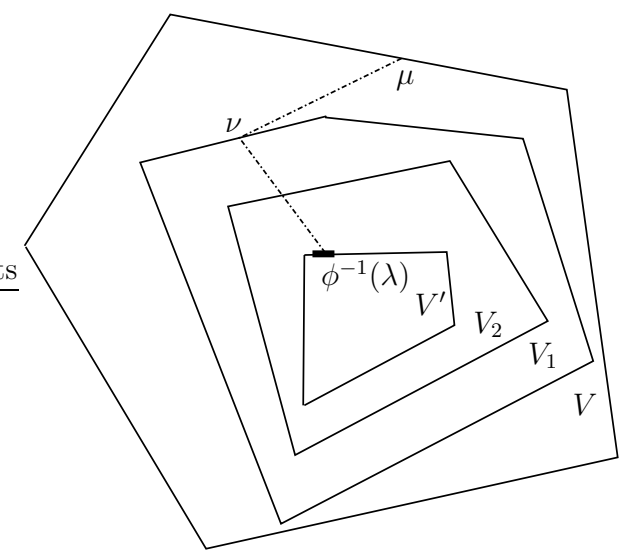

Figure 3: Nested neighbourhoods with possible position of $\mu$ and $\phi^{-1}(\lambda)$.

$\mathcal{M P \mathcal { L }})$ and $\nu \in \mathcal{P} \mathcal{M L} \backslash V_{1}$, the maximal angle of intersection between $\lambda$ and $\phi(\nu)$ is at least $\delta$. If we pick $\varepsilon$ sufficiently small, and we have $\phi(\nu)=\phi(\mu)^{*}$, this violates $\phi(\mu)^{*} \in N_{\varepsilon}^{P T}(\tilde{\lambda})$, for any diagonal extension $\tilde{\lambda}$ of $\lambda$.

\section{Proof of the main theorem}

Our goal is to prove Theorem 1.3, i.e. to verify that $\mathcal{E} \mathcal{L}\left(S_{0,5}\right)$ is homeomorphic to the Nöbeling curve. By Theorem 2.1, in order to do this we must show that $\mathcal{E} \mathcal{L}$ is a Polish space, that it is connected, locally path connected, of dimension 1 , and satisfies the locally finite 1-discs property.

To see that $\mathcal{E} \mathcal{L}$ is separable note that the orbit of any ending lamination under the action of the mapping class group is dense in $\mathcal{E} \mathcal{L}$ (see e.g. Ham06b, Corollary 4.2]). Because the mapping class group is finitely generated, this orbit is countable. Since $\mathcal{E} \mathcal{L}$ is homeomorphic to the Gromov boundary of the curve complex ([Kla99, Theorem 1.3], compare [Ham06, Section 1]), it carries a metric defined using the Gromov product (see Bridson-Haefliger [BH99, Chapter III.H] and Bonk-Schramm [BS00, Section 6]). This metric is complete by [BS00, Proposition 6.2]. Hence $\mathcal{E} \mathcal{L}$ is a Polish space.

By Theorem 4.1, $\mathcal{E} \mathcal{L}$ is connected and locally path connected.

Now we prove that $\mathcal{E} \mathcal{L}$ is of dimension 1 . Since there are paths in $\mathcal{E} \mathcal{L}$ (Theorem 4.1), it is not of dimension 0. In order to prove that $\mathcal{E} \mathcal{L}$ is of dimension at most 1 , we need to check that any point in $\mathcal{E} \mathcal{L}$ has a neighbourhood basis with boundaries of dimension 0 . Let $\mathcal{S}=\left(\left(\mathrm{T}_{k}, \Sigma_{k}\right)\right)_{k}$ be a good partition sequence, guaranteed by Theorem 3.5. Thus, by Corollary 3.9, 
it is enough to prove that the boundary of any $U \in \mathcal{U}(\mathcal{S})$ is of dimension 0 .

The boundary of any $U \in \mathcal{U}(\mathcal{S})$ is contained in a union of boundaries of up to three $U(\tau)$ 's, where $\tau \in \mathrm{T}_{k} \cup \Sigma_{k}$, for some $k$ 's. For $\sigma \in \Sigma_{k}$ the set $U(\sigma)$ is closed (Lemma 3.2(iii)), so that $\operatorname{Fr} U(\sigma) \subset U(\sigma)$. For fixed $k$, all $U(\eta)$ with $\eta \in \mathrm{T}_{k}$ are open and disjoint. Hence, if we denote $X_{k}=\bigcup_{\sigma \in \Sigma_{k}} U(\sigma)$, we have Fr $U(\eta) \subset X_{k}$. By property (Subdivision), for all $K \geq k$ we have $X_{K} \supset X_{k}$. Hence the boundary of any $U \in \mathcal{U}(\mathcal{S})$ is contained in $X_{K}$, for sufficiently large $K$. Thus it is enough to prove that for each $k$ the set $X_{k}=\bigcup_{\sigma \in \Sigma_{k}} U(\sigma) \subset \mathcal{E} \mathcal{L}$ is of dimension 0 .

We obtain a neighbourhood basis for each $\lambda \in X_{k}$ by restricting to $X_{k}$ the sets $U^{\prime} \ni \lambda$ from the open neighbourhood basis $\mathcal{U}(\mathcal{S})$. By Remark 3.8, we may assume that $U^{\prime}=U(\eta)$ or $U^{\prime}=U\left(\eta_{1}\right) \cup U(\sigma) \cup U\left(\eta_{2}\right)$ where $\eta$ or all of $\eta_{1}, \sigma, \eta_{2}$ lie in $\mathrm{T}_{K}$ 's and $\Sigma_{K}$ 's with $K$ 's at least $k$. Since for $K \geq k$ we have $\mathcal{E} \mathcal{L} \backslash X_{K} \subset \mathcal{E} \mathcal{L} \backslash X_{k}$, we get that all $U(\eta)$, with $\eta \in \mathrm{T}_{K}$, are disjoint from $X_{k}$. Hence $U^{\prime}$ is of the form $U\left(\eta_{1}\right) \cup U(\sigma) \cup U\left(\eta_{2}\right)$ and $U^{\prime} \cap X_{k} \subset U(\sigma)$, for some $\sigma \in \Sigma_{K}$.

We now prove that actually we have the equality $U^{\prime} \cap X_{k}=U(\sigma)$. By property (Subdivision), $U(\sigma)$ is contained in some $U(\tau)$, where $\tau \in \mathrm{T}_{k} \cup \Sigma_{k}$. Since $\lambda \in U(\sigma) \subset U(\tau)$ and $\lambda \in X_{k}$, we have $\tau \in \Sigma_{k}$. Hence $U(\sigma) \subset X_{k}$. Summarizing, the restriction of $U^{\prime}$ to $X_{k}$ equals $U(\sigma)$. By Lemma 3.2(iii), $U(\sigma)$ is closed in $\mathcal{E} \mathcal{L}$, hence it is also closed in $X_{k}$. Moreover, $U(\sigma)$ is also open in $X_{k}$, since its complement is a finite union of some disjoint closed $U\left(\sigma^{\prime}\right)$ with $\sigma^{\prime} \in \Sigma_{K}$. Thus the boundary of $U(\sigma)$ in $X_{k}$ is empty, as desired.

In order to finish the proof of Theorem 1.3, it remains to prove the following, which we do in Section 6.

Proposition 5.1. $\mathcal{E} \mathcal{L}\left(S_{0,5}\right)$ satisfies the locally finite 1-discs property.

\section{Universality}

In this section we prove Proposition 5.1, which completes the proof of Theorem 1.3 .

We have to prove that for any family of paths $f_{n}: I \rightarrow \mathcal{E} \mathcal{L}$, where $n \in \mathbb{N}$, and any open cover $\mathcal{U}$ of $\mathcal{E} \mathcal{L}$, there are paths $g_{n}: I \rightarrow \mathcal{E} \mathcal{L}$ such that

(Local finiteness) for each $\lambda \in \mathcal{E} \mathcal{L}$ there is a neighbourhood $U \ni \lambda$ satisfying $g_{n}(I) \cap U=\emptyset$ for sufficiently large $n$,

(Approximation) for each $t \in I, n \in \mathbb{N}$, there is $U \in \mathcal{U}$ such that both $f_{n}(t)$ and $g_{n}(t)$ lie in $U$. 
Because the proof is technically involved, as an illustration we prove the following.

Proposition 6.1. The Nöbeling curve $N_{1}^{3} \subset \mathbb{R}^{3}$ satisfies the locally finite 1-discs property.

Proof. We learned the idea of this proof from Andrzej Nagórko.

We say that a cube $I_{1} \times I_{2} \times I_{3} \subset \mathbb{R}^{3}$ is $m$-diadic if the lengths of all $I_{i}$ equal $\frac{1}{2^{m}}$ and the endpoints of $I_{i}$ lie in $\frac{1}{2^{m}} \mathbb{Z}$.

Assume first, for simplicity, that we are in the special case where there is $m>0$ such that the open cover $\mathcal{U}$ consists of the interiors of unions of pairs of adjacent $m$-diadic cubes. Let $\Gamma \subset \mathbb{R}^{3}$ be the closed set which is the union of all lines parallel to coordinate axes with the fixed coordinate in $\frac{1}{2^{m}} \mathbb{Z}$. In other words $\Gamma$ is the union of 1 -skeleta of all $m$-diadic cubes. Observe that $\Gamma$ is disjoint from $N_{1}^{3}$.

Let $f_{n}: I \rightarrow N_{1}^{3}$ be the family of paths which we want to approximate. We describe the construction of $g_{n}$ for a fixed $n \in \mathbb{N}$.

There is a partition $J_{1} \cup I_{1} \cup \ldots \cup I_{l-1} \cup J_{l}$ of $I$ into closed intervals with disjoint interiors, satisfying the following. There are $m$-diadic open cubes $V_{k}$, where $0 \leq k \leq l$, satisfying $f_{n}\left(I_{k}\right) \subset V_{k}$ and $f_{n}\left(J_{k}\right) \subset \operatorname{int} \overline{V_{k-1} \cup V_{k}}$. Denote the endpoints of $J_{k}$ by $s_{k}, t_{k}$. We can assume $l \geq 1$.

For each pair of adjacent cubes $V_{k-1}, V_{k}$ we denote by $\Gamma_{k} \subset \Gamma$ the square loop which is the intersection of $\Gamma$ with $\partial V_{k-1} \cap \partial V_{k}$. For $A \subset \mathbb{R}^{3}$ and $\delta>0$ we denote by $N_{\delta}(A)$ the open $\delta$-neighbourhood of $A$ in $\mathbb{R}^{3}$.

For each $1<k \leq l$ we choose some $p_{k} \in V_{k-1} \cap N_{\frac{1}{n}}\left(\Gamma_{k}\right)$ satisfying $p_{k} \in N_{1}^{3}$. We put $g_{n}\left(s_{k}\right)=p_{k}$. Analogously, for each $1 \leq \stackrel{\frac{1}{n}}{k}<l$ we choose some $q_{k} \in V_{k} \cap N_{\frac{1}{n}}\left(\Gamma_{k}\right)$ satisfying $q_{k} \in N_{1}^{3}$. We put $g_{n}\left(t_{k}\right)=q_{k}$. We also put $g_{n}(0)=q_{1}$ and $g_{n}(1)=p_{l}$.

For $0<k<l$ we choose paths $h^{I_{k}}$ between $q_{k}$ and $p_{k+1}$ in the open sets $V_{k} \cap N_{\underline{1}}(\Gamma)$. This is possible, since the latter sets are neighborhoods of 1-skeleta of $V_{k}$, hence they are path connected. We define the path $g_{n}$ on $I_{k}$ by slightly perturbing $h^{I_{k}}$ relative the endpoints so that we obtain paths in $N_{1}^{3}$.

Similarly, for $1 \leq k \leq l$ we choose paths $h^{J_{k}}$ between $p_{k}$ and $q_{k}$ in the open sets int $\overline{V_{k-1} \cup V_{k}} \cap N_{\frac{1}{n}}\left(\Gamma_{k}\right)$. The latter sets are path connected because $\Gamma_{k}$ are 1 -spheres. We define the path $g_{n}$ on $J_{k}$ by slightly perturbing $h^{J_{k}}$ relative the endpoints so that we obtain paths in $N_{1}^{3}$.

By construction, paths $g_{n}$ are $\mathcal{U}$-close to $f_{n}$, which means that they satisfy property (Approximation). Moreover, for each $n$ the image of the path $g_{n}$ is contained in $N_{\frac{1}{n}}(\Gamma)$, where $\Gamma$ is a closed set disjoint from $N_{1}^{3}$. This yields property (Local finiteness). This ends the proof in the case of special $\mathcal{U}$. 
In general, we may only assume that $\mathcal{U}$ consists of the interiors of unions of pairs of adjacent $m$-diadic cubes without the assumption that $m$ is fixed. In other words the cubes might be arbitrarily small. However, we can at least assume that no element of $\mathcal{U}$ is properly contained in another one. We also note the property that if two open diadic cubes intersect, then one of them is contained in the other. We define (the "attracting grid") $\Gamma$ as the complement in $\mathbb{R}^{3}$ of the union of all elements of $\mathcal{U}$.

\section{Claim.}

(i) For each pair of adjacent cubes $V_{1}, V_{2}$ with int $\overline{V_{1} \cup V_{2}} \in \mathcal{U}$, the square loop which is the boundary of the common face of $V_{1}$ and $V_{2}$ is contained in $\Gamma$.

(ii) Let $V$ be a maximal (open) cube among all cube pairs from $\mathcal{U}$. Then $\partial V \cap \Gamma$ is connected (and non-empty).

Assertion (i) of the claim follows directly from the maximality assumption on the elements of $\mathcal{U}$. For assertion (ii) observe first that $\partial V$ is a $2-$ sphere. We obtain $\partial V \cap \Gamma$ by removing from $\partial V$ the intersections with elements of $\mathcal{U}$. By maximality assumption on $V$, these elements have the form int $\overline{V_{1} \cup V_{2}}$, where $V_{1} \subset V$ and $V_{2} \subset \mathbb{R}^{3} \backslash V$. Each intersection of such a set with $\partial V$ is an open 2-disc. By the maximality assumption on the elements of $\mathcal{U}$, all those 2 -discs are disjoint. Hence $\partial V \cap \Gamma$ is obtained from a 2 -sphere by removing a disjoint union of open 2-discs, which yields assertion (ii).

We leave it to the reader to verify that the claim allows to perform the same argument as in the special case.

We are now prepared for the following.

Proof of Proposition 5.1. By Theorem 3.5, we may assume that $\mathcal{U} \subset \mathcal{U}(\mathcal{S})$, where $\mathcal{U}(\mathcal{S})$ is the open neighbourhood basis coming from some fixed good partition sequence $\mathcal{S}=\left(\left(\mathrm{T}_{k}, \Sigma_{k}\right)\right)_{k}$ (see Definition 3.7] and Corollary [3.9). Let $\mathcal{U}^{\prime} \subset \mathcal{U}(\mathcal{S})$ be an open cover which is a refinement of $\mathcal{U}$ satisfying the assertion of Lemma 4.4(i). In other words, we require that for any $U^{\prime} \in \mathcal{U}^{\prime}$, there is $U=U\left(U^{\prime}\right) \in \mathcal{U}$ and $\varepsilon=\varepsilon\left(U^{\prime}\right)>0$, so that we have the following. For any $\mu \in V^{\prime}=\Psi\left(U^{\prime}\right)$ with $\phi(\mu) \in \mathcal{A M} \mathcal{L}$, if $\lambda \in \mathcal{E} \mathcal{L}$ and $\phi(\mu)^{*} \in N_{\varepsilon}^{P T}(\tilde{\lambda})$ for some diagonal extension $\tilde{\lambda}$ of $\lambda$, then $\lambda \in U$. Without loss of generality we may assume that whenever $U\left(\eta_{1}\right) \cup U(\sigma) \cup V\left(\eta_{2}\right)$ belongs to $\mathcal{U}^{\prime}$, then also $U\left(\eta_{1}\right)$ and $U\left(\eta_{2}\right)$ belong to $\mathcal{U}^{\prime}$.

We say that a train track $\tau \in \mathrm{T}_{k} \cup \Sigma_{k}$ participates in $\mathcal{U}^{\prime}$, if $U(\tau) \in \mathcal{U}^{\prime}$ or $\tau$ equals $\sigma$ for $U\left(\eta_{1}\right) \cup U(\sigma) \cup U\left(\eta_{2}\right) \in \mathcal{U}^{\prime}$. Let $\mathrm{T}^{\prime} \subset \bigcup_{k} \mathrm{~T}_{k}$ be the family of all complete train tracks $\eta$ participating in $\mathcal{U}^{\prime}$ with maximal $V(\eta)$ with respect 
to inclusion. In other words, we take all complete train tracks participating in $\mathcal{U}^{\prime}$ and remove those $\eta$ whose $V(\eta)$ is properly contained in some $V\left(\eta^{\prime}\right)$, where $\eta^{\prime}$ is also participating in $\mathcal{U}^{\prime}$. Note that by property (Subdivision) $V(\eta)$ and $V\left(\eta^{\prime}\right)$ can only intersect if one is contained in the other. We denote the family of $U(\eta)$ over $\eta \in \mathrm{T}^{\prime}$ by $\mathcal{U}\left(\mathrm{T}^{\prime}\right)$. Denote $\mathcal{V}\left(\mathrm{T}^{\prime}\right)=\Psi\left(\mathcal{U}\left(\mathrm{T}^{\prime}\right)\right)$. Since $V(\eta)$ were required to be maximal, the elements of $\mathcal{V}\left(\mathrm{T}^{\prime}\right)$ are pairwise disjoint. Hence the elements of $\mathcal{U}\left(\mathrm{T}^{\prime}\right)$ are also pairwise disjoint.

Let $\Sigma^{\prime} \subset \bigcup_{k} \Sigma_{k}$ be the family of all nearly complete train tracks $\sigma$ participating in $\mathcal{U}^{\prime}$ with maximal $V(\sigma)$ with respect to inclusion, among all $V(\tau)$ with $\tau$ participating in $\mathcal{U}^{\prime}$. We denote the family of $U(\sigma)$ over $\sigma \in \Sigma^{\prime}$ by $\mathcal{U}\left(\Sigma^{\prime}\right)$ and we put $\mathcal{V}\left(\Sigma^{\prime}\right)=\Psi\left(\mathcal{U}\left(\Sigma^{\prime}\right)\right)$. The elements of $\mathcal{V}\left(\Sigma^{\prime}\right)$ are pairwise disjoint and disjoint from the elements of $\mathcal{V}\left(\mathrm{T}^{\prime}\right)$. Hence the elements of $\mathcal{U}\left(\Sigma^{\prime}\right)$ are also pairwise disjoint and disjoint from the elements of $\mathcal{U}\left(\mathrm{T}^{\prime}\right)$.

Let $\Gamma \subset \mathcal{P} \mathcal{M L}$ be the closed set which is the complement of the union of all sets in $\mathcal{V}^{\prime}=\Psi\left(\mathcal{U}^{\prime}\right)$. We have $\Gamma \cap \mathcal{M P} \mathcal{M} \mathcal{L}=\emptyset$.

\section{Claim 1.}

(i) For any $\sigma \in \Sigma^{\prime}$, we have $\partial V(\sigma) \subset \Gamma$.

(ii) For any $\eta \in T^{\prime}$ the set $\partial V(\eta) \cap \Gamma$ is connected and non-empty.

\section{Proof of Claim 1.}

(i) Let $\mu \in \partial V(\sigma)$. If $\mu \notin \Gamma$, then there is $V^{\prime} \in \mathcal{V}^{\prime}$ with $\mu \in V^{\prime}$. Since $V^{\prime}$ is open in $\mathcal{P} \mathcal{M L}$, it intersects $V(\sigma)$. The set $V^{\prime}$ is of the form $V^{\prime}=V(\eta)$ or $V^{\prime}=V\left(\eta_{1}\right) \cup V\left(\sigma^{\prime}\right) \cup V\left(\eta_{2}\right)$. Thus $V(\sigma)$ intersects $V(\tau)$, for $\tau$ equal to one of $\eta, \eta_{i}, \sigma^{\prime}$. Since $\sigma \in \Sigma^{\prime}$, we have $V(\tau) \subset V(\sigma)$. Hence $\tau$ is a nearly complete train track, and therefore $V^{\prime}=V\left(\eta_{1}\right) \cup V\left(\sigma^{\prime}\right) \cup V\left(\eta_{2}\right)$ where $\sigma^{\prime}$ is equal to $\tau$. Since $\sigma \in \Sigma^{\prime}$, we have $V(\sigma) \supset V\left(\sigma^{\prime}\right)$. By hypothesis $\mu$ is outside of $V(\sigma)$, hence it lies in $V\left(\eta_{i}\right)$ for some $i$. But then $V\left(\eta_{i}\right)$ intersects $V(\sigma)$ and like before we get $V\left(\eta_{i}\right) \subset V(\sigma)$, which is a contradiction.

(ii) First note that $\partial V(\eta)$ is a 2-sphere. $\partial V(\eta)$ is disjoint from any $V\left(\eta^{\prime}\right)$, for $\eta^{\prime}$ participating in $\mathcal{U}^{\prime}$ : otherwise $V\left(\eta^{\prime}\right)$ intersects $V(\eta)$ and by maximality of $V(\eta)$ (since $\eta \in \mathrm{T}^{\prime}$ ) we have $V\left(\eta^{\prime}\right) \subset V(\eta)$, which is a contradiction. Hence, if for some $V^{\prime} \in \mathcal{V}^{\prime}$ the intersection $\partial V(\eta) \cap V^{\prime}$ is non-empty, then $V^{\prime}=V\left(\eta_{1}\right) \cup V(\sigma) \cup V\left(\eta_{2}\right)$, where $\sigma \in \Sigma^{\prime}$, and $\partial V(\eta) \cap V^{\prime} \subset V(\sigma)$. Moreover, since $V^{\prime}$ is open, we have that $V(\sigma)$ or one of $V\left(\eta_{i}\right)$ intersects $V(\eta)$. Since $\sigma \in \Sigma^{\prime}$, this must be one of $V\left(\eta_{i}\right)$. Because $\eta \in \mathrm{T}^{\prime}$, we then have $V\left(\eta_{i}\right) \subset V(\eta)$. In particular, $P(\sigma) \subset \partial V\left(\eta_{i}\right) \subset P(\eta)$. Again, since $\sigma \in \Sigma^{\prime}$ we have $P(\sigma) \subset \partial V(\eta)$. 
Summarizing, for any $V^{\prime} \in \mathcal{V}^{\prime}$ we have $\partial V(\eta) \cap V^{\prime}=V(\sigma)$, for some $\sigma \in \Sigma^{\prime}$, which is an open 2-disc. Hence $\partial V(\eta) \cap \Gamma$ is obtained from the 2 -sphere $\partial V(\eta)$ by removing a (possibly infinite) union of disjoint open 2 -discs.

Let $f_{n}: I \rightarrow \mathcal{E} \mathcal{L}$, where $n \in \mathbb{N}$, be the family of paths which we want to approximate. We now independently construct the paths $g_{n}$. To this end, we fix $n \in \mathbb{N}$ and note the following.

Claim 2. There is a partition $I_{0} \cup J_{1} \cup I_{1} \cup \ldots \cup J_{l} \cup I_{l}$ of $I$ into closed intervals with disjoint interiors, with possibly empty $I_{0}, I_{l}$, satisfying the following (see Figure 4).

- $f_{n}\left(I_{k}\right) \subset U_{k}^{\prime}$, for some $U_{k}^{\prime} \in \mathcal{U}\left(\mathrm{T}^{\prime}\right) \subset \mathcal{U}^{\prime}$, if $I_{k}$ is non-empty, where $0 \leq k \leq l$.

- $f_{n}\left(J_{k}\right) \subset \hat{U}_{k}$, where $\hat{U}_{k}=U\left(\eta_{k}^{1}\right) \cup U\left(\sigma_{k}\right) \cup U\left(\eta_{k}^{2}\right) \in \mathcal{U}^{\prime}$ with $\sigma_{k} \in \Sigma^{\prime}$. Moreover, for $j=k-1, k$ there is $i$ such that $U\left(\eta_{k}^{i}\right) \subset U_{j}^{\prime}$, if $I_{j}$ is non-empty, where $1 \leq k \leq l$.

Proof of Claim 2. For the proof it is convenient to introduce the following terminology. We call an element $U$ of $\mathcal{U}(\mathcal{S})$ a vertex block if it is of the form $U(\eta)$ for some complete train track $\eta$. We call the other elements of $\mathcal{U}(\mathcal{S})$ edge blocks.

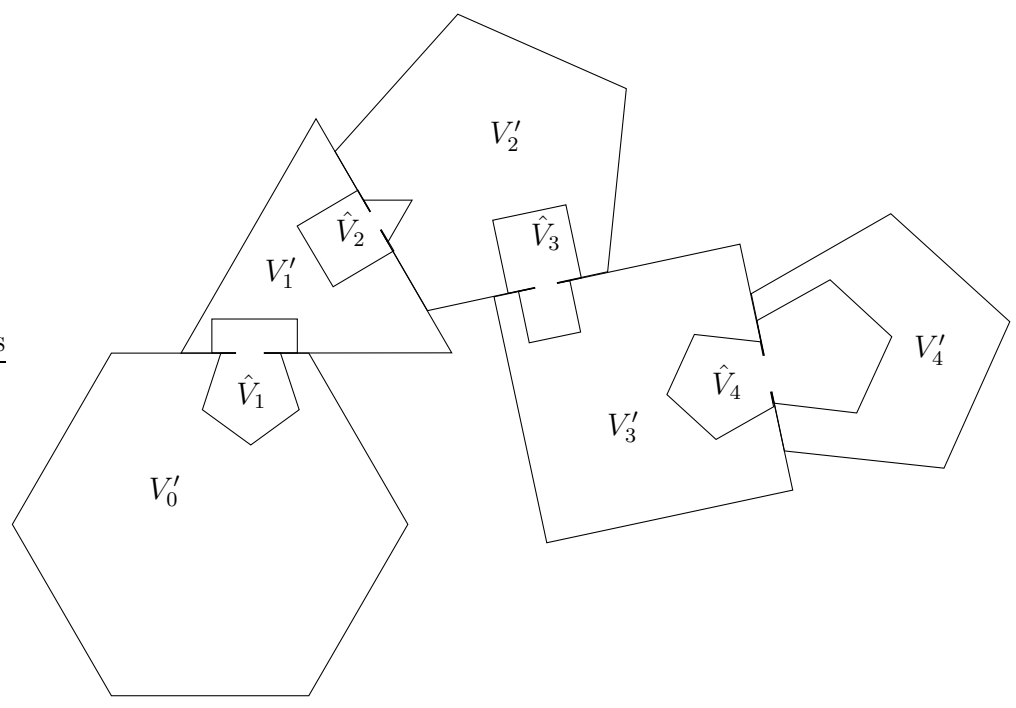

Figure 4: Combinatorics of vertex blocks and edge blocks. 
Consider now the family $\mathcal{Y}$, which is the union of all vertex blocks in $\mathcal{U}\left(\mathrm{T}^{\prime}\right) \subset \mathcal{U}^{\prime}$ and edge blocks $U\left(\eta^{1}\right) \cup U(\sigma) \cup U\left(\eta^{2}\right) \in \mathcal{U}^{\prime}$ with $\sigma \in \Sigma^{\prime}$, where we pick only one such set for each $\sigma$. Observe that $\mathcal{Y}$ forms an open cover of $\mathcal{E} \mathcal{L}$. By compactness of $I$, a finite subset of $\mathcal{Y}$ covers $f_{n}(I)$. In particular, there is a partition $\mathcal{I}$ of $I$ into finitely many nontrivial closed intervals with disjoint interiors so that each interval is mapped into a set of $\mathcal{Y}$. Observe that two consecutive intervals in $\mathcal{I}$ cannot be mapped into different vertex blocks, since the latter are disjoint. Moreover, if two consecutive intervals $I_{-}, I_{+} \in \mathcal{I}$ are mapped into edge blocks $U\left(\eta_{-}^{1}\right) \cup U\left(\sigma_{-}\right) \cup U\left(\eta_{-}^{2}\right), U\left(\eta_{+}^{1}\right) \cup U\left(\sigma_{+}\right) \cup U\left(\eta_{+}^{2}\right) \in \mathcal{U}^{\prime}$, then we have the following. Since $\sigma_{-} \neq \sigma_{+}$, we have that $f_{n}\left(I_{-} \cap I_{+}\right)$lies in, say, $U\left(\eta_{-}^{1}\right) \cap U\left(\eta_{+}^{1}\right)$, hence $U\left(\eta_{-}^{1}\right)$ and $U\left(\eta_{+}^{1}\right)$ are contained in the same vertex block $U^{\prime} \in \mathcal{U}\left(\mathrm{T}^{\prime}\right)$. We can then represent $I_{-} \cup I_{+}=I_{-}^{\prime} \cup J \cup I_{+}^{\prime}$ with $I_{-}^{\prime} \subset I_{-}, I_{+}^{\prime} \subset I_{+}$and $f_{n}(J) \subset U^{\prime}$, where $J$ is nontrivial. To conclude this discussion, we can assume that the intervals in $\mathcal{I}$ are mapped alternatively into vertex blocks and edge blocks of $\mathcal{Y}$. Furthermore, observe that for each pair of consecutive intervals in $\mathcal{I}$ mapped by $f_{n}$ to $U(\eta) \in \mathcal{Y}$ and $U\left(\eta^{1}\right) \cup$ $U(\sigma) \cup U\left(\eta^{2}\right) \in \mathcal{Y}$ there is some $i$ with $U\left(\eta^{i}\right) \subset U(\eta)$. This gives rise to $I_{k}, J_{k}$ as required.

From now on we fix the objects and the notation as in Claim 2. Before we describe the construction of the path $g_{n}$, note that to guarantee property (Approximation) it suffices that $g_{n}$ satisfies the following two properties.

(Approximation i) For each $0 \leq k \leq l$ such that $I_{k}$ is non-empty we have $g_{n}\left(I_{k}\right) \subset U\left(U_{k}^{\prime}\right)$.

(Approximation ii) For each $1 \leq k \leq l$ we have $g_{n}\left(J_{k}\right) \subset U\left(\hat{U}_{k}\right)$.

At this point we can finally define the path $g_{n}$. Denote $V_{k}^{\prime}=\Psi\left(U_{k}^{\prime}\right)$ and $\hat{V}_{k}=\Psi\left(\hat{U}_{k}\right)$. If $l=0$, we choose any point $p \in V_{0}^{\prime} \cap N_{\frac{1}{n}}(\Gamma)$ satisfying $\phi(p) \in \mathcal{E} \mathcal{L}$. This is possible since the open set $V_{0}^{\prime} \cap N_{\frac{1}{n}}(\Gamma)^{n}$ is non-empty by Claim 1 (ii) and $\mathcal{M P \mathcal { M }}$ is dense in $\mathcal{P} \mathcal{M} \mathcal{L}$. We put $g_{n}(I) \equiv p$, which obviously satisfies properties (Approximation i) and (Approximation ii).

From now on we assume $l \geq 1$. Denote the endpoints of $J_{k}$ by $s_{k}, t_{k}$. First we claim that for any $1 \leq k \leq l$ with $s_{k} \neq 0$ the open set $V_{k-1}^{\prime} \cap \hat{V}_{k} \cap$ $N_{\frac{1}{n}}\left(\partial V\left(\sigma_{k}\right)\right)$ is non-empty. Indeed, there is an $i$ satisfying $V\left(\eta_{k}^{i}\right) \subset V_{k-1}^{\prime}$. Then we have $\partial V\left(\sigma_{k}\right) \subset \partial V\left(\eta_{k}^{i}\right) \subset \bar{V}_{k-1}^{\prime}$. Hence $\partial V\left(\sigma_{k}\right)$ is contained in the closure of $V_{k-1}^{\prime} \cap \hat{V}_{k}=V\left(\eta_{k}^{i}\right)$ and the claim follows.

Thus for each $1 \leq k \leq l$ with $s_{k} \neq 0$ we can choose some $p_{k} \in V_{k-1}^{\prime} \cap \hat{V}_{k} \cap$ $N_{\frac{1}{n}}\left(\partial V\left(\sigma_{k}\right)\right)$ satisfying $\phi\left(p_{k}\right) \in \mathcal{E} \mathcal{L}$. We put $g_{n}\left(s_{k}\right)=\phi\left(p_{k}\right)$. Analogously, for each $1 \leq k \leq l$ with $t_{k} \neq 1$ we choose some $q_{k} \in V_{k}^{\prime} \cap \hat{V}_{k} \cap N_{\frac{1}{n}}\left(\partial V\left(\sigma_{k}\right)\right)$ 


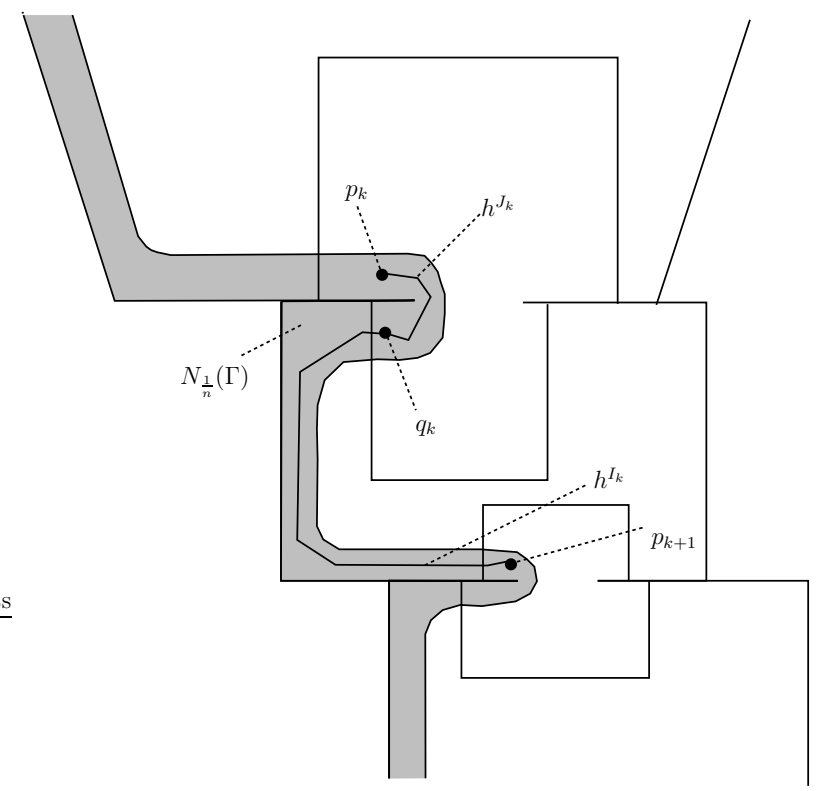

Figure 5: Construction of approximating paths attracted by $\Gamma$.

satisfying $\phi\left(q_{k}\right) \in \mathcal{E} \mathcal{L}$. We put $g_{n}\left(t_{k}\right)=\phi\left(q_{k}\right)$. By Claim 1(i) all $p_{k}, q_{k}$ lie in $N_{\frac{1}{n}}(\Gamma)$.

If $s_{1}=0$ (i.e. if $I_{0}$ is empty), then we put $g_{n}(0)=q_{1}$, otherwise we put $g_{n}\left(I_{0}\right) \equiv p_{1}$. Analogously, if $t_{l}=1$ (i.e. if $I_{l}$ is empty), then we put $g_{n}(1)=p_{l}$, otherwise we put $g_{n}\left(I_{l}\right) \equiv q_{l}$.

For $0<k<l$, we choose some PL almost filling paths $h^{I_{k}}$ between $q_{k}$ and $p_{k+1}$ in the sets $V_{k}^{\prime} \cap N_{\frac{1}{n}}(\Gamma)$ (see Figure 5). This is possible, since the latter sets are open and path connected by Claim 1(ii). To each $h^{I_{k}}$ we apply Lemma 4.3 with $\varepsilon=\varepsilon_{n}^{\prime}=\min \left\{\varepsilon\left(U_{k}^{\prime}\right), \frac{1}{n}\right\}$ (and any $\delta$ ). We obtain paths $g_{n}^{I_{k}}: I_{k} \rightarrow \mathcal{E} \mathcal{L}$ with endpoints $\phi\left(q_{k}\right), \phi\left(p_{k+1}\right)$, such that the image of $g_{n}^{I_{k}}$ lies in $U\left(U_{k}^{\prime}\right)$. We define $g_{n}$ on $I_{k}$ to be equal to $g_{n}^{I_{k}}$, which gives property (Approximation i).

Similarly, for $1 \leq k \leq l$ we choose some PL almost filling paths $h^{J_{k}}$ between $p_{k}$ and $q_{k}$ in the sets $\hat{V}_{k} \cap N_{\frac{1}{n}}\left(\partial V\left(\sigma_{k}\right)\right)$ (also see Figure 5). This is possible since the latter sets are open and path connected (because $\partial V\left(\sigma_{k}\right)$ are 1 -spheres). To each $h^{J_{k}}$ we apply Lemma 4.3 with $\varepsilon=\hat{\varepsilon}_{n}=\min \left\{\varepsilon\left(\hat{U}_{k}\right), \frac{1}{n}\right\}$. We obtain paths $g_{n}^{J_{k}}: J_{k} \rightarrow \mathcal{E} \mathcal{L}$ with endpoints $\phi\left(p_{k}\right), \phi\left(q_{k}\right)$, such that the image of $g_{n}^{J_{k}}$ lies in $U\left(\hat{U}_{k}\right)$. We define $g_{n}$ on $J_{k}$ to be equal $g_{n}^{J_{k}}$, which gives property (Approximation ii).

This concludes the construction of the paths $g_{n}: I \rightarrow \mathcal{E} \mathcal{L}$. By the discussion above they satisfy property (Approximation). 
It remains to verify property (Local finiteness). Let $\lambda_{0} \in \mathcal{E} \mathcal{L}$. Let $V \in$ $\mathcal{V}(\mathcal{S})$ be a neighbourhood of $\phi^{-1}\left(\lambda_{0}\right)$ such that its closure is disjoint from $\Gamma$ (guaranteed by Theorem 3.5(Fineness)). Put $U=\phi(V \cap \mathcal{M P \mathcal { M }} \mathcal{L}) \in \mathcal{U}(\mathcal{S})$. Let $U^{\prime} \subset U$ and $\varepsilon>0$ be as in the assertion of Lemma 4.4(ii) applied to $U$ and $\lambda_{0}$. For sufficiently large $n$ we have that $V$ is outside $N_{\frac{1}{n}}(\Gamma)$ and $\frac{1}{n} \leq \varepsilon$. Then both $\hat{\varepsilon}_{n}$ and $\varepsilon_{n}^{\prime}$ are smaller than $\varepsilon$, and therefore the image of $g_{n}$ is outside $U^{\prime}$.

\section{References}

[BS00] M. Bonk and O. Schramm, Embeddings of Gromov hyperbolic spaces, Geom. Funct. Anal. 10 (2000), no. 2, 266-306.

[Bow85] P. L. Bowers, Dense embeddings of sigma-compact, nowhere locally compact metric spaces, Proc. Amer. Math. Soc. 95 (1985), no. 1, 123-130.

[BH99] M. R. Bridson and A. Haefliger, Metric spaces of non-positive curvature, Grundlehren der Mathematischen Wissenschaften [Fundamental Principles of Mathematical Sciences], vol. 319, Springer-Verlag, Berlin, 1999.

[Cur85] D. W. Curtis, Boundary sets in the Hilbert cube, Topology Appl. 20 (1985), no. $3,201-221$.

[Dug58] J. Dugundji, Absolute neighborhood retracts and local connectedness in arbitrary metric spaces, Compositio Math. 13 (1958), 229-246 (1958).

[Eng78] R. Engelking, Dimension theory, North-Holland Publishing Co., Amsterdam, 1978. Translated from the Polish and revised by the author; North-Holland Mathematical Library, 19.

[Gab09] D. Gabai, Almost filling laminations and the connectivity of ending lamination space, Geom. Topol. 13 (2009), no. 2, 1017-1041.

[Ham06a] U. Hamenstädt, Train tracks and the Gromov boundary of the complex of curves, Spaces of Kleinian groups, London Math. Soc. Lecture Note Ser., vol. 329, Cambridge Univ. Press, Cambridge, 2006, pp. 187-207.

[Ham06b] _ Geometric properties of the mapping class group, Problems on mapping class groups and related topics, Proc. Sympos. Pure Math., vol. 74, Amer. Math. Soc., Providence, RI, 2006, pp. 215-232.

[Ham09] , Geometry of the mapping class groups. I. Boundary amenability, Invent. Math. 175 (2009), no. 3, 545-609.

[KLT97] K. Kawamura, M. Levin, and E. D. Tymchatyn, A characterization of 1dimensional Nöbeling spaces, Proceedings of the 12th Summer Conference on General Topology and its Applications (North Bay, ON, 1997), 1997, pp. 155174 .

[Kla99] E. Klarreich, The boundary at inifinity of the curve complex and the relative Teichmüller space (1999), available at http://www.msri.org/people/members/klarreic/curvecomplex.ps. 
[LS08] C. Leininger and S. Schleimer, Connectivity of the space of ending laminations (2008), preprint, available at arXiv:0801.3058.

[LMS08] C. Leininger, M. Mj, and S. Schleimer, Universal Cannon-Thurston maps and the boundary of the curve complex (2008), preprint, available at arXiv:0808.3521.

[Luo00] F. Luo, Automorphisms of the complex of curves, Topology 39 (2000), no. 2, 283-298.

[MM99] H. A. Masur and Y. N. Minsky, Geometry of the complex of curves. I. Hyperbolicity, Invent. Math. 138 (1999), no. 1.

[Mos03] L. Mosher, Train track expansions of measured foliations (2003), unpublished manuscript, available at http://andromeda.rutgers.edu/〜mosher/arationality_03_12_28.pdf.

[Nag06] A. Nagórko, Characterization and topological rigidity of Nöbeling manifolds (2006), submitted, available at arXiv:0602574 PhD thesis.

[PH92] R. C. Penner and J. L. Harer, Combinatorics of train tracks, Annals of Mathematics Studies, vol. 125, Princeton University Press, Princeton, NJ, 1992.

[Thu80] W. P. Thurston, The geometry and topology of three-manifolds (1980), available at http:msri.org/publications/books/gt3m/. 